\title{
Non-Rayleigh Acoustic Scattering Characteristics of Individual Fish and Zooplankton
}

\author{
Timothy K. Stanton, Dezhang Chu, and D. Benjamin Reeder
}

\begin{abstract}
It has long been known that the statistical properties of acoustic echoes from individual fish can have non-Rayleigh characteristics. The statistical properties of echoes from zooplankton are generally less understood. In this study, echoes from individual fish and zooplankton from a series of laboratory measurements from the past decade are investigated. In the experiments, acoustic echoes from various individual organisms were measured over a wide range of frequencies and orientations, typically in $1^{\circ}-3^{\circ}$ increments. In the analysis in this paper, the echoes from most of those measurements are grouped according to ranges of orientation, which correspond to typical orientation distributions of these organisms in the natural ocean environment. This grouping provides a distribution of echo values for each range of orientation. This approach, in essence, emulates a field experiment whereby distributions of echoes would be recorded for different distributions of orientations of the organisms. For both the fish and zooplankton data, there are conditions under which the echoes are strongly non-Rayleigh distributed. In some cases, the distribution is quantitatively connected to the physics of the scattering process while, in other cases, the connection is described qualitatively. Exploitation of the animal-specific statistics for classification purposes is suggested.
\end{abstract}

Index Terms-Acoustic scattering, echo statistics, fish, zooplankton.

\section{INTRODUCTION}

A CTIVE acoustic methods have long been used to rapidly and remotely survey marine organisms such as fish and zooplankton. A critical element in the interpretation of the echo data is understanding the acoustic scattering characteristics of the organisms. Models of the scattering, used in combination with ground truth data such as net tows, can help to provide estimates of meaningful biological parameters, such as numerical density, size, and taxa of the organisms.

A common approach toward acoustically assessing the distribution of organisms is to use a model or measure of the average acoustic scattering by individual organisms. The average scattering value is combined with the volume-scattering strength of

Manuscript received July 17, 2003; revised December 23, 2003. This work was supported by the U.S. Navy, by the U.S. Office of Naval Research, by the National Oceanographic and Atmospheric Administration, and by the Massachusetts Institute of Technology/Woods Hole Oceanographic Institution (WHOI) Joint Graduate Education Program in Oceanographic Engineering. This is Woods Hole Oceanographic Institution Contribution 11064.

T. K. Stanton and D. Chu are with the Department of Applied Ocean Physics and Engineering, Woods Hole Oceanographic Institution, Woods Hole, MA 02543-1053 USA (e-mail: tstanton@ whoi.edu).

D. B. Reeder was with the Department of Applied Ocean Physics and Engineering, Woods Hole Oceanographic Institution, Woods Hole, MA 02543-1053 USA. He is now with the Department of Oceanography, Naval Postgraduate School, Monterey, CA 93943 USA.

Digital Object Identifier 10.1109/JOE.2004.828208 the water column to obtain average quantities such as numerical density of organisms, as listed above. Although this is a very useful and robust approach, it also loses much statistical information through the averaging process.

There is information in the statistical characteristics of the individual echoes related to important features of the organism, such as size, anatomical group, and orientation distribution. For example, in the research reviewed by [1], the echo-amplitude probability density function (pdf) of the echoes from individual fish is narrow (Gaussian-like) for calm fish and broad (Rayleighlike) for active fish whose orientation varied significantly. In the research of [2], the echo-amplitude pdf was shown to be dependent upon the size of zooplankton; the small-sized zooplankton produced pdfs that were narrow and Gaussian-like, while the larger zooplankton produced Rayleigh-like pdfs. MacLennon and Menz [3] have proposed a method to extract the targetstrength/length relationship from the echo pdf of individual fish. In the research of [4], the statistical properties of the frequency spectrum of broad-band echoes from zooplankton had characteristics specific to the particular anatomical group to which the zooplankton belonged.

While most of the above studies have shown, at least qualitatively, that the statistical properties of the echoes from individual organisms can be non-Rayleigh under important conditions, the studies were generally not controlled relative to the orientation of the organisms. Specifically, orientation plays a key role in both the overall scattering levels as well as the statistical behavior of the echoes. The orientation distribution of organisms varies with animal behavior. Furthermore, the orientation of the organisms relative to the sonar changes with deployment geometry of the acoustic system (e.g., downlooking versus sidelooking). Given the variety of organism behavior and deployment geometries, there is a need to understand the dependence of the statistical properties of the echoes from individual organisms upon the distribution of their orientation relative to the sonar.

This paper investigates the orientation dependence of the statistical properties of acoustic echoes from individual fish and zooplankton. This analysis makes use of the results of a series of measurements that have been conducted over the past decade of the acoustic scattering by the individuals over a wide range of frequencies and orientations. Generally, the orientation was varied in $1^{\circ}-3^{\circ}$ increments in one or two planes of rotation. From these data, the echoes are grouped and binned into histograms for different ranges of orientations. These ranges correspond to important orientation distributions of the organisms in the natural oceanic environment. The statistical characteristics of the echoes are shown to vary with orientation distribution and have non-Rayleigh properties under important conditions. 

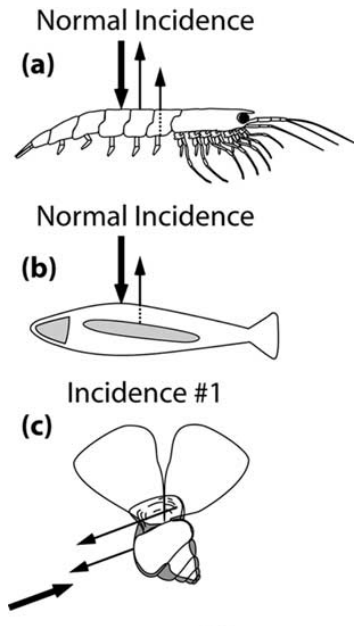

(d)

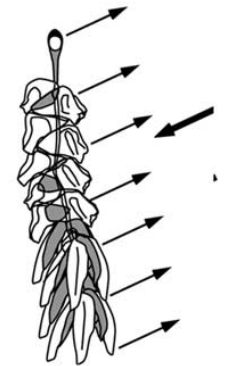

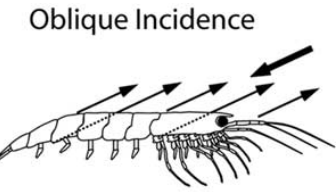

Oblique Incidence
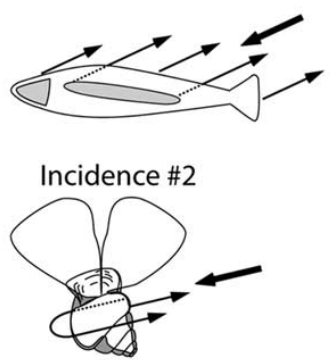

Fig. 1. Schematic of scattered rays by various types of organisms; shrimp, swimbladder-bearing fish, periwinkle, and siphonophore in (a)-(d), respectively. The fish in (b) contains a swimbladder, as illustrated by the shaded elongated feature in its interior and the siphonophore in (d) contains a gas inclusion, as indicated by the top-most scattering feature. In (c), incidence \#1 corresponds to the case in which the scattering is dominated by the echo from the front interface and inside of opercular opening of the periwinkle and Incidence \#2 corresponds to when the scattering is dominated by the echo from the front interface and a partially circumnavigating Lamb wave.

In all cases, the statistics of the echoes are related to the physics of the scattering at least qualitatively, if not also quantitatively. For example, the echoes are strongly non-Rayleigh when a small number of scattering features (traceable to the anatomy of the organisms) dominate the scattering.

The results of this study apply directly to high-resolution sonars in which the echoes from the individual organisms are resolved. For the cases in which they are not resolved, the echoes from the group echo would be further complicated by the additive effects of the individual echoes. In addition to the influence of the physics of the scattering as described in this paper, the group echo would also depend upon the number of organisms within a resolution cell as well as their average separation, degree of spatial heterogeneity (i.e., patchiness), and size distribution (see, for example, [5] and [6]).

\section{Echo Statistics of AN $N$-RAy SCATtering Process}

The acoustic scattering by marine organisms is complex and only under certain conditions can the echo statistics be simply related to the scattering physics. Generally, in the geometric scattering region (i.e., wavelengths much shorter than any dimension of the target), the scattering can be described through a ray representation of the scattering (Fig. 1). In this region, each ray is associated with the scattering by a particular physical feature, such as an edge, outer surface, or an organ, and will have an asso-

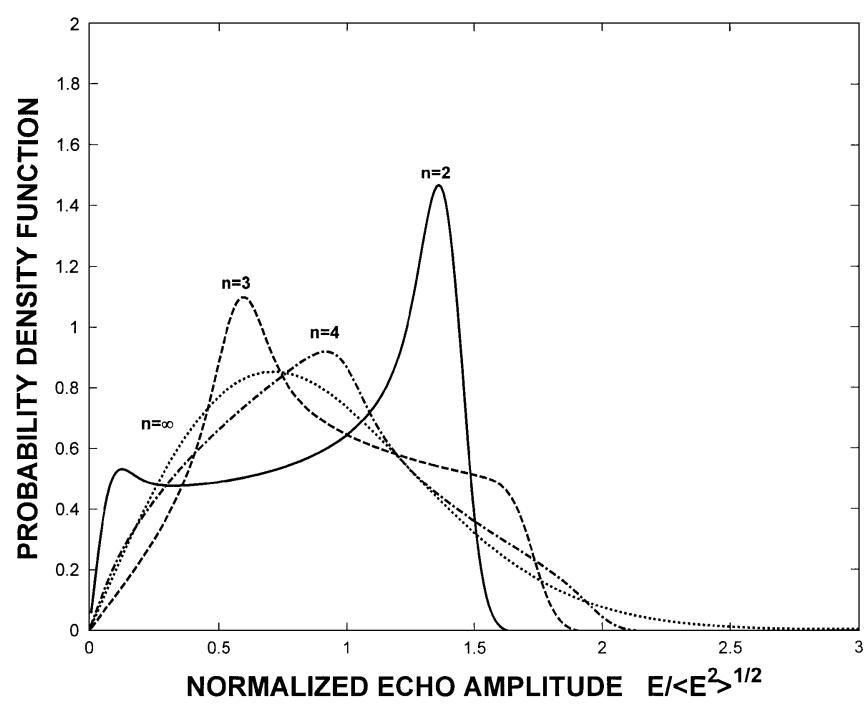

Fig. 2. Envelope pdfs from sums of $n$ random phase sinusoids, where $n=2-4$ and infinity. Curves based on a combination of equations from [14] and [15].

ciated amplitude and phase, depending on the size, shape, orientation, position, and material property of the feature, as well as the wavelength of the sound. The total scattered field will be comprised of the sum of these rays. As the orientation of the organism changes, so will the amplitude and phase of the rays.

Depending on the type of organism and scattering condition (such as orientation and frequency), the scattering can be modeled as one or more rays. The resultant echo-amplitude statistics will vary significantly, depending on how many rays contribute significantly to the scattering (Fig. 2). Generally, a small number of rays will result in a non-Rayleigh pdf and a large number of rays will result in a Rayleigh pdf. For example, a single ray will result in the delta-function pdf. Two rays will have a bimodal pdf associated with it. As the number of rays increases toward six and beyond, the pdf will increasingly resemble the Rayleigh pdf (Fig. 2).

In certain special cases, there will be a single strong ray superimposed upon many weaker rays. The resultant pdf is the Rice pdf, which tends to a Gaussian (and, eventually, a delta function) for high ratios of energy of the strong ray to collective energy of the weaker rays [7]. For low ratios, it tends to the Rayleigh. The shape of the pdf varies continuously, from that of the Rayleigh pdf to that of the delta-function pdf, for the full range of the ratios.

\section{LABORATORY MEASUREMENTS AND DATA PROCESSING}

All acoustic backscattering measurements were performed in tanks using live individual animals during the period 19932000. Two series of measurements were performed at sea with a tank on the deck of a ship, using live freshly caught zooplankton. The remainder of the measurements were performed on land. Over the course of these studies, more than 100 organisms were studied. Four representative cases will be presented here, involving a low-aspect-ratio hard elastic shelled organism (periwinkle), two elongated high-aspect-ratio species (fish and shrimp), and an elongated gelatinous organism with a low-aspect-ratio gas inclusion (siphonophore), all of which are illustrated in Fig. 1 


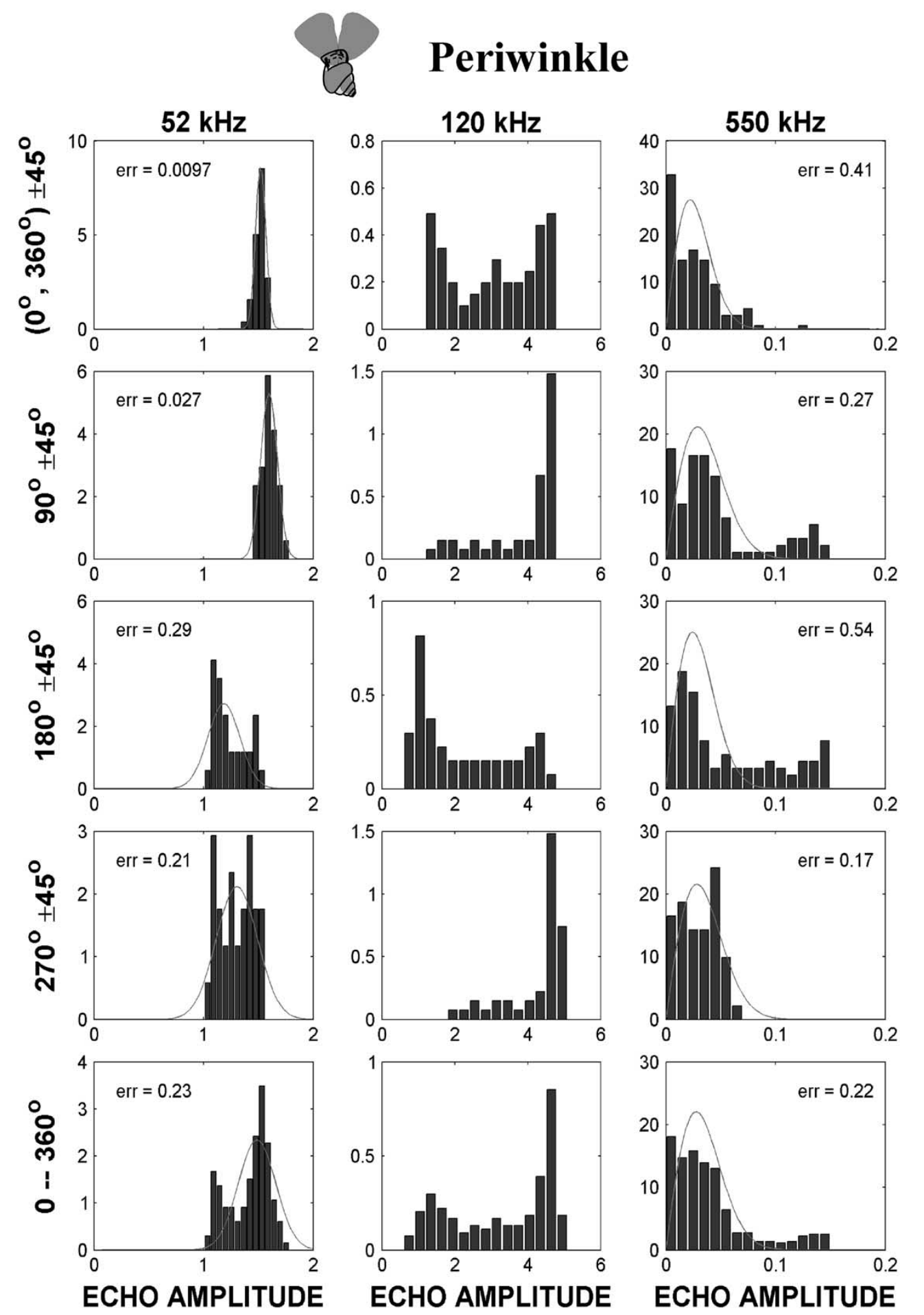

Fig. 3. Echo-amplitude histograms (solid) using laboratory backscattering data from an individual periwinkle shell (diameter of a sphere with the same volume is $4.6 \mathrm{~mm}$ ). Histograms were formed by grouping the echo data from ranges of orientations and from data (animal 97-1) described in [10]. Values of $k a$ range from 0.5 at $52 \mathrm{kHz}$ to 5 at $550 \mathrm{kHz}$. The Gaussian (curves in the left column) and Rayleigh (curves in the right column) pdfs were fit to the data using a least-squares approach. The error from the approach is given in an upper corner and $0^{\circ}$ corresponds to the acoustic wave incident upon the apex of the periwinkle (point opposite "winged" section shown in Fig. 1).

The zooplankton measurements involved tanks that were $1.5-\mathrm{m}$ deep and either $2.4 \mathrm{~m}$ in diameter or $2.4 \mathrm{~m}$ wide by $3.7 \mathrm{~m}$ long. The dimensions of the cubic tank for the fish measurements were $6 \mathrm{~m}$ per side. Each setup involved use of one pair of closely spaced transducers per frequency or frequency band. One transducer was used as a transmitter while the other was used as a receiver. Commercial pulse-echo electronics were used to transmit and receive the echoes. A computer-controlled signal generator was used to create the various signals that were fed into a power amplifier that, in turn, applied a high-voltage signal to the transmitting transducer. The received echo was first amplified by a preamplifier, then filtered before being 


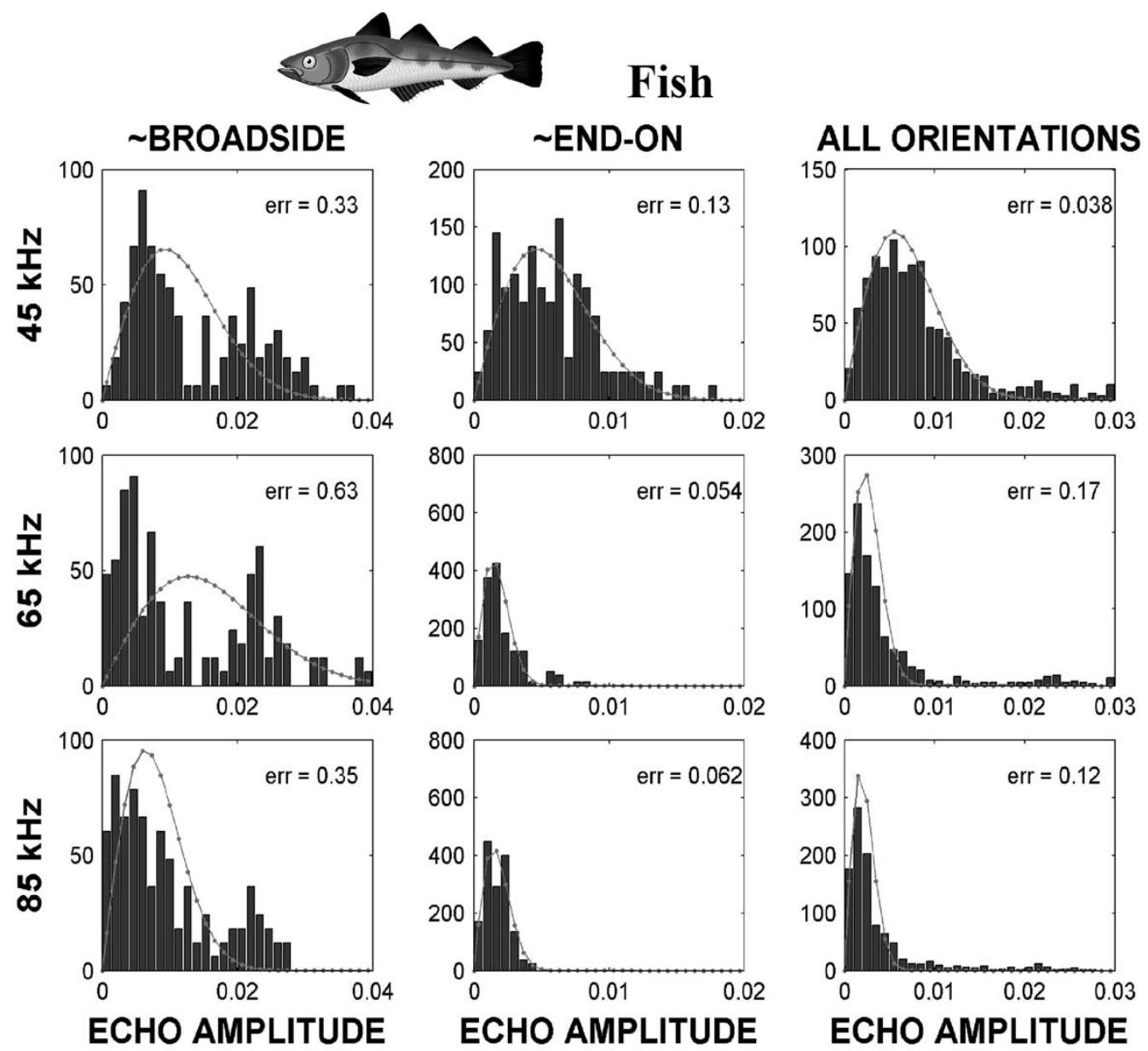

Fig. 4. Echo-amplitude histograms (solid) using laboratory backscattering data from a live individual Alewife (caudal lengths $=22 \mathrm{~cm}$ ). Broadside corresponds to dorsal and ventral incidence $\pm 15^{\circ}$, end-on corresponds to tail- and head-on incidence $\pm 15^{\circ}$, and "all orientations" corresponds to $0^{\circ}-360^{\circ}$. Rayleigh pdfs (curves) were fit in the same manner as described in the caption to Fig. 3. The values of $k a$ are approximately $1-2$, while $k L / 2$ is about 7-13, where $a$ (=0.5 cm) is the cross-sectional radius of the swimbladder and $L(=7.5 \mathrm{~cm})$ is its length. The histograms were formed from data (Alewife 15) described in [12].

digitized and displayed on a digital oscilloscope that, in turn, transferred the data to the computer for display and storage. Gated sine waves were used for the narrow-band transducers, while linear frequency-modulation signals ("chirps") were applied to the broad-band transducers that typically had an octave bandwidth. All organisms were suspended by thin monofilament lines, which had negligibly small echoes. The organisms in the ship-based measurements were suspended by a simple tether system with little or no control over orientation (orientation was monitored with a video camera). In the land-based measurements, the tether system was more complex and the orientation of the organisms was controlled through the use of a computer-controlled stepper motor. The motor stepped through $1^{\circ}-3^{\circ}$ increments per ping over all angles of orientation in two planes of rotation. Prior to each measurement and before the organism was placed in the acoustic beam, the reverberation of the tank was coherently measured and stored. Once the organism was placed in the beam, the reverberation was subtracted in real time from the echo, so that only the echo from the organism remained (plus random noise). This process of subtraction has proven to be relatively robust over the decade of experiments and has routinely removed echoes from the rigid and fixed structures with rejection ratios of tens of decibels. Details of the experiments, including calibration, are given in [8]-[12].
In order to emulate the statistical behavior of the echoes that might be expected in the natural ocean environment,echoes were grouped within ranges of orientations. These ranges correspond to the orientation distributions that may be encountered in the ocean. In all cases, the echo envelope (magnitude of backscattering amplitude) was analyzed. Once grouped, the echoes were binned to form an echo histogram for that particular distribution of orientations. For example, for an up- or downlooking echosounder and a hovering or swimming animal, the orientations near normal incidence were grouped together. For a sidelooking sonar, all orientations $\left(0^{\circ}-360^{\circ}\right)$ were used, since the orientations would most likely be, over a long enough period of time, randomly and uniformly distributed. For organisms migrating quickly up- or downward, the orientations near end-on were grouped together (up- or downlooking echo sounder).

\section{RESULTS}

The echo-amplitude histograms showed significant variation with respect to orientation distribution, animal type, and acoustic frequency (Figs. 3-6). In many of the histograms, attempts were made to fit the Rayleigh pdf to the distributions. In certain other cases, attempts were also made to fit Gaussian and Rice pdfs to the data. For each organism, there were conditions under which the histograms were non-Rayleigh. Errors ("err") between the 


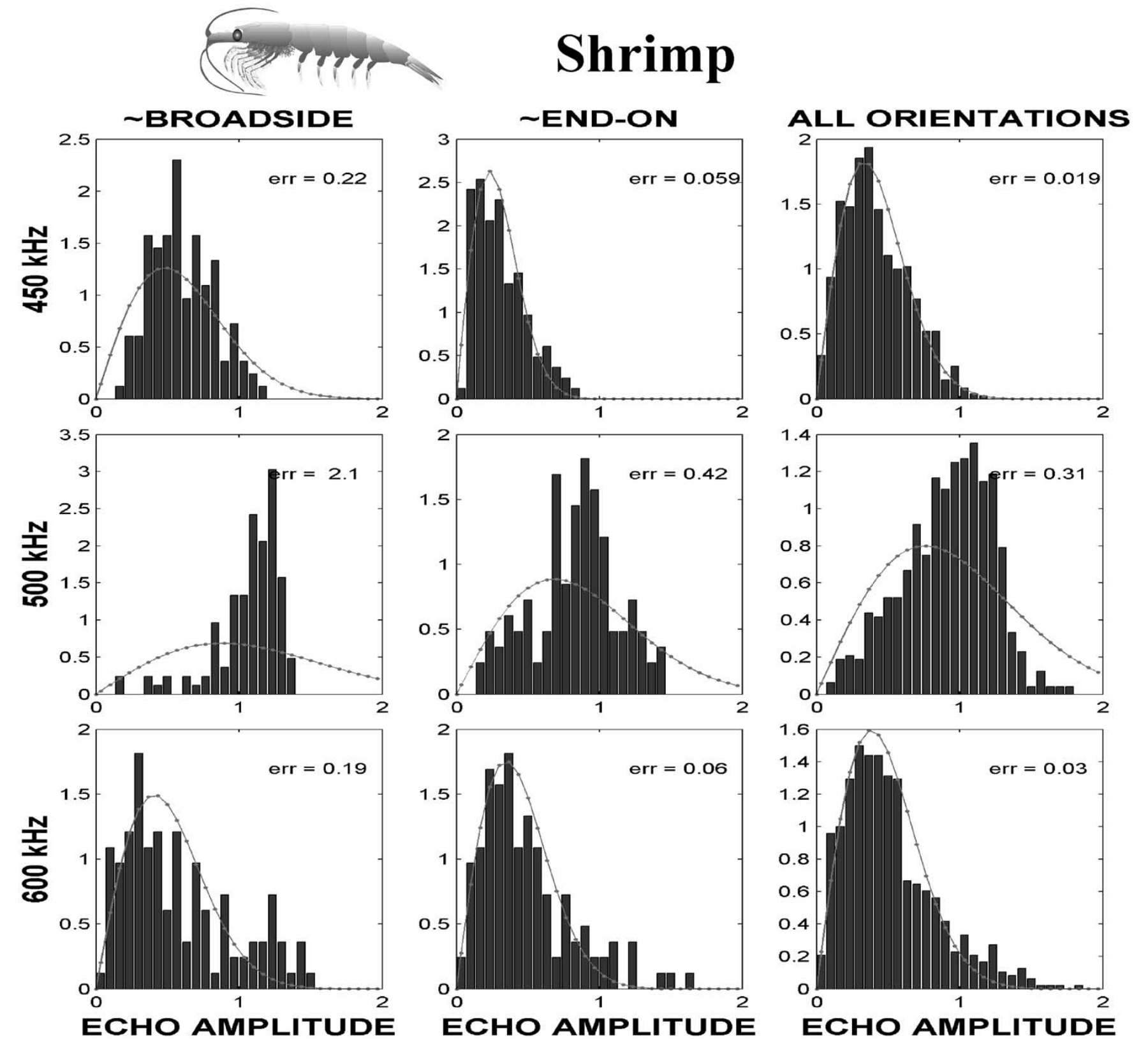

Fig. 5. Echo-amplitude histograms (solid) using laboratory backscattering data from a live individual decapod shrimp (length $=3.0 \mathrm{~cm}$ ). The histograms were formed from data (Animal 6) described in [11]. The values of $k a$ and $k L / 2$ are in the approximate ranges of 3-4 and 30-40, respectively, where $a(=1.5 \mathrm{~mm})$ is the cross-sectional radius of the shrimp. Details on the histograms, orientations, and Rayleigh pdf curves given in the caption to Fig. 4 , with the exception that the plane of rotation was the lateral plane and "broadside" is the side aspect.

data and theoretical curves were calculated the same way for all plots for intercomparison and were displayed in some of the panels. The errors were calculated by normalizing the sum of the square of the difference between the data (histogram bars) and theoretical curve by the sum of the square of the corresponding points on the theoretical curve.

For the rounded organisms (periwinkles), histograms over a wide range of frequencies are presented $(52-550 \mathrm{kHz}$; Fig. 3). At the lowest frequency $(52 \mathrm{kHz})$, the histograms are narrow for all orientation distributions and sometimes are Gaussian-like. As the frequency increases, the distributions widen to an intermediate distribution at $120 \mathrm{kHz}$ and approach the Rayleigh pdf at $550 \mathrm{kHz}$.

For the elongated organisms (fish and shrimp), the echoes generally displayed Rayleigh-like behavior when the orienta- tions were centered near end-on incidence (middle columns, Figs. 4 and 5) and for the data involving all orientations (third columns, Figs. 4 and 5), with the exception of the $500-\mathrm{kHz}$ data for the shrimp. For orientations near broadside, the distributions were generally non-Rayleigh for the two organisms (first columns, Figs. 4 and 5), with the possible exception of the $600-\mathrm{kHz}$ data for the shrimp. Also, the histograms for the fish appeared to be bimodal for these orientations (first column, Fig. 4), with two peaks occurring in each histogram at relative echo amplitudes of approximately 0.006 and 0.022 .

In the case of the gas-bearing zooplankton (siphonophore), orientation of this very flexible organism was not controlled. In contrast to the above studies, the statistical characteristics of the echoes were studied both with and without the gas inclusion 


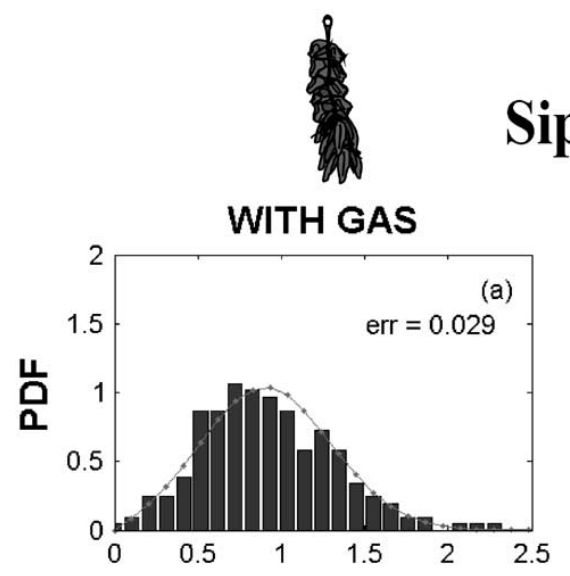

\section{Siphonophore}
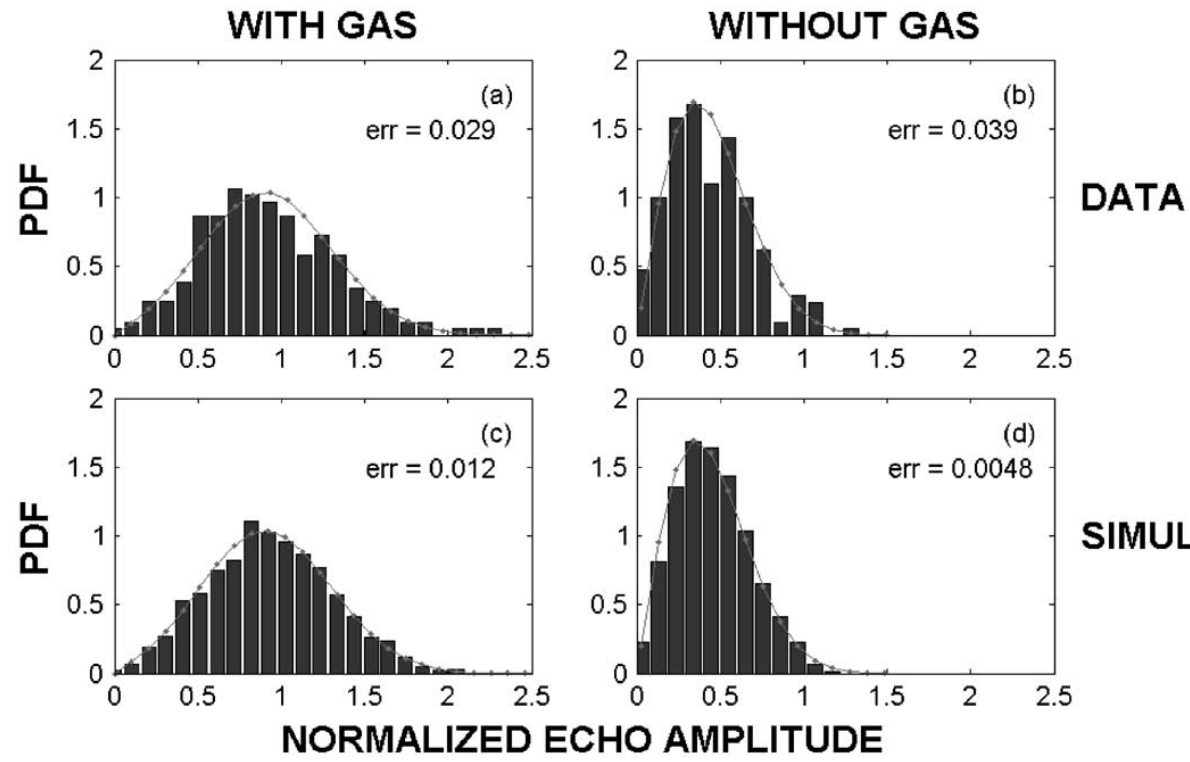

Fig. 6. Echo-amplitude histograms (upper row, solid) using laboratory backscattering data from a live individual siphonophore (body length $=4.8 \mathrm{~cm}$; diameter of a sphere with the same volume of gas is $0.85 \mathrm{~mm}$ ). Acoustic frequency is the $560-\mathrm{kHz}$ component of the broad-band echo. The histograms were formed from a series of pings in which the organism was freely moving while on a tether in the acoustic beam (there was no control over the orientation). The left column corresponds to data involving the whole organism, while the right column corresponds to the organism with the gas inclusion removed through dissection. The bottom row corresponds to simulations: Right plot—many random-phase rays to describe the tissue scattering without gas. Left plot—-the tissue rays added to a single constant ray to describe the scattering by the tissue and gas. The solid curves in all plots are the Ricean pdfs, while in the left column it has a Gaussian-like appearance and in the right column it has become the Rayleigh pdf. The value of $k L / 2$ is about 55 (from [9]).

present. The measurements show that the echoes from the whole organism are non-Rayleigh. Once the gas inclusion is removed and only the elongated body tissue remained, then the echoes were Rayleigh distributed (Fig. 6).

\section{INTERPRETATION IN TERMS OF SCATTERING PHYSICS}

The statistics of the observed echoes from the various organisms can be related, at least qualitatively, to the dominant scattering processes associated with them. Because of the difference in types of organisms (e.g., low- and high-aspect ratios) and types of data collected (a wide range of frequencies for some, but not all, organisms), the interpretation of the data is organized as follows. All combinations of a wide range of frequencies and orientation distributions for the low-aspect-ratio target (periwinkle), three different orientation distributions at an intermediate-to-high set of frequencies for the high-aspect-ratio targets (fish and shrimp), and the special case of the gas-bearing zooplankton (siphonophore) with and without the gas (through dissection).

\section{A. Low-Aspect-Ratio Target}

Given the rounded shape of the periwinkle, the analysis is organized according to frequency regime, rather than orientation. The full frequency range is explored, ranging from low frequency, where $k a=0.5$, to high frequency, where $k a=5$ (the intermediate frequency case involves values of $k a=1.2$ ). Here, $k=2 \pi / \lambda$ ( $\lambda$ is the acoustic wavelength) is the acoustic wavenumber and $a$ is the average radius of the target.
1) Low Acoustic Frequency-Non-Rayleigh: The low frequency $(52-\mathrm{kHz})$ scattering by the periwinkle produced echo pdfs that were consistently narrow and sometimes Gaussian-like (left column, Fig. 3). As shown in [10], the scattering at this frequency by this animal does not depend strongly on orientation. Any grouping of orientation angles will thus result in little variation of scattering levels, as demonstrated in Fig. 3. The explanation of these effects is straightforward, as the wavelengths $(28 \mathrm{~mm})$ are greater than the dimensions of the target $(5 \mathrm{~mm})$. Specifically, $k a=0.5$; thus, the scattering is in the upper portion of the Rayleigh $(k a \ll 1)$ scattering region. In this region, the phase difference between the scattering from any parts of the target is relatively small. Changes in orientation will not result in significant changes in phase of the scattering by any parts. Thus, the target acts principally as a single scattering feature with a narrowly distributed range of echo levels.

2) Intermediate Acoustic Frequency-Non-Rayleigh: As the frequency is increased for the periwinkle, other scattering phenomena become important (Fig. 3, middle column). In this region, rays from different scattering features contribute significantly to the scattering. As demonstrated in [10], at least two rays contribute to the scattering and these rays have phases that depend upon orientation, size, and acoustic wavelength. Depending on the orientation, there is some combination of rays from the front interface, a circumnavigating Lamb wave, and back surface inside the opercular opening. The histograms in this midfrequency region are generally bimodal, broadly consistent with two-ray scattering (Fig. 2). 
3) High Acoustic Frequency-Tending to Rayleigh: Once the frequency increases even further than in the above case, the echo statistics for the periwinkle tend to Rayleigh, although in the examples shown in Fig. 3 (right column), the pdfs have yet to reach the Rayleigh pdf. It is hypothesized that at these higher frequencies, the smaller features (edges, roughness of the shell) become more of a factor and more than two rays can be contributing to the scattering in this case. As a result, the object becomes more of a diffuse scatterer in this region.

\section{B. High-Aspect-Ratio Targets}

Given the strong dependence of the scattering (and associated echo statistics) upon orientation, the analysis is organized according to several important orientation distributions-ones centered around end-on and normal incidence, as well as one in which the orientation distribution is uniformly distributed across all orientations. The frequency range is in the intermediate range relative to cross-sectional radius (according to the definition in the previous section), where $k a$ is around 1-2 for the fish and 3-4 for the shrimp, and $a$ is the cross-sectional radius of the swimbladder of the fish and body of the shrimp. However, the frequencies are high relative to length, as $k L / 2$ is about 7-13 for the swimbladder and 30-40 for the shrimp, where $L$ is the length of the swimbladder or body of the shrimp.

1) End-On Incidence-Rayleigh: The echoes near end-on incidence of the two elongated targets are generally Rayleigh distributed (middle columns, Figs. 4 and 5, except for the $500-\mathrm{kHz}$ data with the shrimp). Since $k L$ is much greater than unity, the phase of the scattered acoustic signal varies rapidly along the length of the target for any given orientation near end-on. When the orientation changes, those relative variations change significantly. In this case, the scattering can be modeled in terms of many rays of random phase and amplitude radiating from various parts of the body. The fact that the echo statistics are Rayleigh indicates that there are well more than six rays that contribute significantly to the scattering (Fig. 2). This is consistent with the scattering geometry; for near end-on incidence, there is no single dominant feature. There mostly are irregularities along the length of the targets. Since the wavelengths are small as compared with the overall dimensions of the targets, then the phases of the rays radiating from those irregularities will tend to be random. The changing orientation will change the set of phases into a new set of random values.

2) Normal Incidence-Non-Rayleigh: For the cases involving normal incidence for the fish and shrimp, the pdfs are generally non-Rayleigh (first column, Figs. 4 and 5, except for the $600-\mathrm{kHz}$ data with the shrimp). As shown in previous studies, under these conditions, there are a small number of scattering features that dominate the scattering. In the case of the fish, the swimbladder has been shown to dominate the scattering. Since the swimbladder is elongated, there still are phase variations along the length and related directional characteristics of the scattering. For the shrimp, the front and back interface have been shown to dominate the scattering, hence resulting in two rays that contribute significantly to the scattering.

None of the histograms from the fish and shrimp in the normal incidence case resemble a one-, two-, or three-ray pdf. Clearly, the scattering is much more complicated than what a simple, equal-amplitude ray description could describe for the histograms. The histogram at $500 \mathrm{kHz}$ for the shrimp case most deviates from the Rayleigh pdf. This frequency coincides approximately with a null in the pattern of target strength versus frequency. The destructive interference at this frequency apparently contributes to the non-Rayleigh characteristics. Given the bimodal nature of some of the histograms for both fish and shrimp, there is the suggestion that two or three rays may be contributing to the scattering in a significant way (Fig. 2). Since there is not a consistent resemblance between these pdfs and the $n=2$ and $n=3$ plots of Fig. 2, certainly the scattering process is more complicated than that. In each case, there are other parts of the bodies that contribute to the scattering. Although those parts may not dominate the average level of scattering, they can possibly contribute enough to alter the statistical behavior of the scattering.

3) All Orientations-Rayleigh: Once all of the orientations are included in the echo-amplitude histograms, the echoes generally tend to be even closer to Rayleigh than in the end-on case. It is hypothesized that, in this case, there are so many rays contributing to the scattering from each of the different orientations that, as in the central limit theorem, the distribution tends to Rayleigh.

\section{Spherical Gas Inclusion-Non-Rayleigh}

In the case of the siphonophore, there are two distinct scattering phenomena taking place. One involves the nearly spherical gas inclusion, which produces a significant echo and whose scattering is not strongly dependent upon orientation. The other involves the gelatinous tissue that contains many facets. The tissue portion of the siphophonore produces a Rayleigh-distributed echo at high frequencies [Fig. 6(b) and (d)]. Once the gas inclusion is present, the echoes are non-Rayleigh [Fig. 6(a) and (c)]. In fact, it has been shown that the distribution is Rice distributed, which is due to the sum of two types of rays: one a delta-function-distributed ray (from the gas) and the other a Rayleigh-distributed ray [from the tissue; curves in Fig. 6(a) and (c)].

\section{SUMMARY AND CONCLUSION}

As a result of the various groupings of data, the echo-amplitude distributions from individual organisms were shown to be strong functions of orientation distribution, as well as animal type and shape and acoustic frequency. The distributions can be related, at least qualitatively, in terms of the scattering features of the targets. At low frequencies, the phase of the acoustic signal is relatively constant across the target, which acts as a single scatterer. In this case, the echo amplitude is narrowly distributed and non-Rayleigh. As the frequency is increased, the phase across the target begins to change significantly. In this region, certain scattering features may dominate and elongation of the target also becomes a factor. For end-on orientation or distributions involving most or all of the angles, there can be many scattering features that contribute to the scattering resulting in a Rayleigh-distributed echo. However, in certain cases, such as for distributions centered near normal incidence, there may be a small number of scattering features, which results in a non-Rayleigh distribution. Finally, in the special case 
of siphonophores in which there exists a small gas inclusion in the irregular gelatinous tissue, the gas produces a constant level echo superimposed upon the Rayleigh-distributed echoes from the tissue, resulting in a (non-Rayleigh) Rice-distributed echo.

The analysis in this paper is based on experiments in which the shape was held relatively constant for the fish and shrimp. However, for free-swimming organisms in the ocean, bending of the body throughout a series of pings could cause a departure of the echo statistics from those reported in this paper. For example, in [13], the backscattering by finite cylinders was demonstrated to depend strongly on the degree to which the cylinders were bent. If the shape of the fish and shrimp changes in addition to the orientation, there will be most likely a broadening of their echo-amplitude pdfs over those reported herein. Such changes are not anticipated for the pdfs associated with the other two organisms as the periwinkle is rigid and the siphonophore was allowed to flex freely throughout the experiment.

As discussed in Section I, the statistical characteristics of echoes from resolved targets can be exploited for target discrimination and classification. The results in this study show that the echoes from the various zooplankton and fish are strongly non-Rayleigh under important conditions. It is possible that the dependences of the statistical parameters of the echoes upon important biological parameters can be used to help to differentiate between organisms of different sizes, orientation distributions, and gross anatomical features, such as those discussed in [1]-[4]. For example, the shape of the pdf is shown to change from Gaussian-like for small $k a$ to Rayleigh-like for high $k a$ (Fig. 3), hence providing a measure of size of the object, which can be deduced from the transition frequency. Also, observing pdfs with a Rice distribution at high acoustic frequencies would suggest the presence of a gas-bearing zooplankton.

In conclusion, the acoustic scattering by marine organisms is complex and description of the echo-amplitude statistics of the resolved individuals is correspondingly complex. The scattering by the individuals can be described, at least qualitatively, through individual rays caused by various dominant scattering features. The number and strength of the rays depend strongly upon size, shape, orientation, and material properties of the target, as well as acoustic frequency. If the number of rays is high enough, such as at high frequencies in some cases, then the echoes tend to be Rayleigh distributed. However, there are important conditions, such as at low-to-intermediate frequencies and/or at normal incidence, under which the echoes are non-Rayleigh.

\section{ACKNOWLEDGMENT}

The authors would like to thank S. Barkley of the Woods Hole Oceanographic Institution (WHOI), Woods Hole, MA, for preparing the manuscript of this paper.

\section{REFERENCES}

[1] T. K. Stanton and C. S. Clay, "Sonar echo statistics as a remote sensing tool: Volume and sea floor," IEEE J. Oceanic Eng., vol. OE-11, pp. 79-96, Jan. 1986.

[2] P. H. Wiebe, C. H. Greene, T. K. Stanton, and J. Burczynski, "Sound scattering by live zooplankton and micronekton: Empirical studies with a dual beam acoustical system," J. Acoust. Soc. Amer, vol. 88, pp. 2346-2360, 1990.
[3] D. N. MacLennan and A. Menz, "Interpretation of in situ target-strength data," Int. Council for the Exploration of the Sea J. Mar. Sci., vol. 53, pp. 233-236, 1996.

[4] L. V. M. Traykovski, T. K. Stanton, P. H. Wiebe, and J. F. Lynch, "Model based covariance mean variance classification techniques: Algorithm development and applications to the acoustic classification of zooplankton," IEEE J. Ocean. Eng., vol. 23, pp. 344-364, Oct. 1998.

[5] V. V. Ol'shevskii, Characteristics of Sea Reverberation. New York: Consultants Bureau, 1967.

[6] T. C. Gallaudet and C. P. de Moustier, "High-frequency volume and boundary acoustic backscatter fluctuations in shallow water," J. Acoust. Soc. Amer, vol. 114, pp. 707-725, 2003.

[7] S. O. Rice, "Mathematical analysis of random noise," in Selected Papers on Noise and Stochastic Processes, N. Wax, Ed. New York: Dover, 1954, pp. 133-294.

[8] T. K. Stanton, D. Chu, P. H. Wiebe, L. Martin, and R. L. Eastwood, "Sound scattering by several zooplankton groups I: Experimental determination of dominant scattering mechanisms," J. Acoust. Soc. Amer., vol. 103 , pp. 225-235, 1998

[9] T. K. Stanton, D. Chu, and P. H. Wiebe, "Sound scattering by several zooplankton groups II: Scattering models," J. Acoust. Soc. Amer., vol. 103, pp. 236-253, 1998.

[10] T. K. Stanton, D. Chu, P. H. Wiebe, R. L. Eastwood, and J. D. Warren, "Acoustic scattering by benthic and planktonic shelled animals," $J$. Acoust. Soc. Amer., vol. 108, pp. 535-550, 2000.

[11] A. C. Lavery, T. K. Stanton, D. E. McGehee, and D. Chu, "Three-dimensional modeling of acoustic backscattering from fluid-like zooplankton," J. Acoust. Soc. Amer., vol. 111, pp. 1197-1210, 2002.

[12] D. B. Reeder, J. M. Jech, and T. K. Stanton, Broadband acoustic backscatter and high-resolution morphology of fish: Measurement and modeling, in J. Acoust. Soc. Amer., to be published.

[13] T. K. Stanton, "Sound scattering by cylinders of finite length III: Deformed cylinders," J. Acoust. Soc. Amer., vol. 86, pp. 691-705, 1989.

[14] R. Barakat, "First-order statistics of combined random sinusoidal waves with applications to laser speckle patterns," Optica. Acta., vol. 21, pp. 903-921, 1974.

[15] J. K. Jao and M. Elbaum, "First-order statistics of a non-Rayleigh fading signal and its detection," Proc. IEEE, vol. 66, pp. 781-790, 1978.

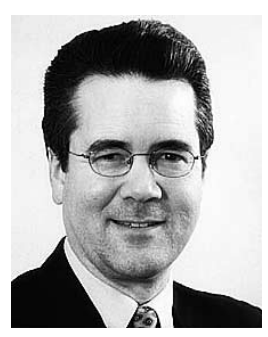

Timothy K. Stanton received the B.S. degree from Oakland University, Rochester, MI, in 1974 and the M.S. and Ph.D. degrees from Brown University, Providence, RI, in 1977 and 1978, respectively, all in physics. His undergraduate research involved experiments in solid-state acoustics with Dr. N. Tepley as advisor and his graduate research involved nonlinear acoustics measurements and instrumentation development with Dr. R. Beyer as advisor.

From 1978 to 1980, he was a Senior Engineer with the Submarine Signal Division, Raytheon Company, Portsmouth, RI, where he was engaged in research, development, and testing of acoustic systems. From 1980 to 1988, he was a Member of the Scientific Staff, Department of Geology and Geophysics, University of Wisconsin, Madison, where he conducted various theoretical, laboratory, and field studies in acoustical oceanography with Dr. C. Clay. From 1988 to the present, he has been a Member of the Scientific Staff, Department of Applied Ocean Physics and Engineering, Woods Hole Oceanographic Institution (WHOI), Woods Hole, MA. From 1997 to 2001, he was Chair of the department and currently is a Senior Scientist. He currently is working in collaboration with colleagues in the development of acoustic scattering models of marine organisms through various theoretical and laboratory methods and is applying the methods to field surveys. In addition to his research, he is part of the Massachusetts Institute of Technology (MIT), Cambridge, and WHOI Joint Graduate Education Program and teaches acoustic scattering theory. Overall, he has published papers covering the areas of nonlinear acoustics, acoustics instrumentation, fiber-optic hydrophones, and acoustic scattering by volumetric objects, sea floor, sea surface, and the underside of sea ice. He has served as Associate Editor for the Journal of the Acoustical Society of America and as a Guest Editor for a special issue of Deep Sea Research.

Dr. Stanton has also served as a Guest Editor for a special issue of the IEEE JOURNAL OF OCEANIC ENGINEERING. He is a Fellow of the Acoustical Society of America and is a Member of The Oceanography Society. In 1985, he was awarded the A. B. Wood Medal for Distinguished Contributions to Underwater Acoustics. 


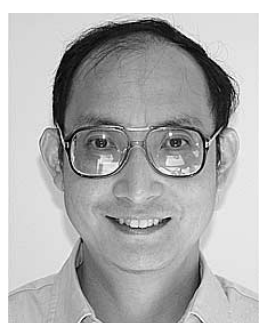

Dezhang Chu received the B.A. degree in electrical engineering from China University of Geosciences, Wuhan, China, in 1982 and the Ph.D. degree in geophysics from the University of Wisconsin, Madison, in 1989.

He was a Postdoctoral Scholar from 1989 to 1990 and a Postdoctoral Investigator from 1990 to 1991, both with the Woods Hole Oceanographic Institution (WHOI), Woods Hole, MA. He currently is a Research Specialist with the Department of Applied Ocean Physics and Engineering, WHOI. His research interests include acoustic scattering, wave propagation and reverberation in shallow water, acoustical signal processing, and sea-floor characterization.

Dr. Chu is a Fellow of the Acoustical Society of America.

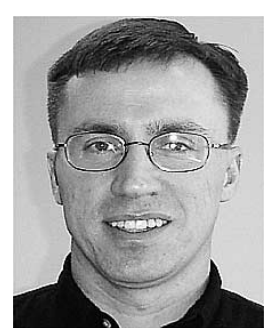

D. Benjamin Reeder received the B.S. degree in physics from Clemson University, Clemson, SC, in 1988 and the Ph.D. degree in oceanographic engineering from the Massachusetts Institute of Technology (MIT), Cambridge, and Woods Hole Oceanographic Institution (WHOI), Woods Hole, MA, Joint Program in Oceanography and Oceanic Engineering in 2002. His graduate research involved underwater acoustic scattering theory and experimentation, with Dr. T. K. Stanton as advisor.

From 1988 to 1993, he was a Nuclear Engineering Officer on the nuclear-powered submarines, USS Tecumseh (SSBN-628), USS George Washington Carver (SSBN-656), and USS Ulysses S. Grant (SSBN631), qualifying as a Submarine Warfare Officer in 1991 and as a Naval Nuclear Engineer in 1992. From 1993 to 1997, he served as a Naval Oceanographer with the Naval Submarine Group Nine, Bangor, WA, and the Naval Pacific Meteorology and Oceanography Center West/Joint Typhoon Warning Center, Guam. He was a Meteorology and Oceanography Officer from June 2002 to November 2003 aboard the USS Tarawa (LHA-1), deploying to the Arabian Gulf during Operation Iraqi Freedom. He still is on active duty as a Lieutenant Commander in the U.S. Navy and currently serves as Assistant Professor of Oceanography and Underwater Acoustics at the Naval Postgraduate School (NPS), Monterey, $\mathrm{CA}$. He is currently conducting research in underwater acoustics in collaboration with colleagues at WHOI and NPS.

Dr. Reeder is a Member of the Acoustical Society of America. 